\title{
Data Dependence Result and Stability of Jungck- Picard-S Iteration Scheme for Approximating Fixed Point of Jungck-Contraction Mappings
}

\author{
Zeana Zaki Jamil ${ }^{1}$, Assma Khaldoon Abdullateef ${ }^{2}$ \\ University of Baghdad-College of Science-Department of Math-Baghdad-Iraq
}

\begin{abstract}
In this paper, we study the stability of a Jungck-Picard-S iteration method when applied to a pair of Jungck-contraction mappings under certain condition. Furthermore, we prove a data dependence result for fixed point of a pair of Jungck-contraction mappings under certain condition with the help of the Jungck-Picard-S iteration method.
\end{abstract}

Keywords: Jungck-Picard-S iterative Scheme, Jungck-contraction mappings, Data dependence, Stability

\section{Introduction and Preliminaries}

Fixed point theory is concerned with investigating a wide variety of issues such as the existence (and uniqueness) of fixed points, the construction of fixed points, etc. One of these themes is data dependency of fixed points. Data dependency of fixed points has been the subject of research in fixed point theory for some time now, and data dependence research is an important theme in its own right. Several authors had made contributions to the study of data dependence of fixed points such as Berinde [2] and others.

In the study of iterations, it is also important to examine their stability. The concept of stability was introduced by Harder [5], Harder and Hicks [6], [7] and roughly speaking of a fixed point iteration procedure is numerically stable if small modification in the initial data involved in the computation process will produce a small influence on the computed value of the fixed point.

In this paper, we establish data dependence result of JungckPicard-S iterative scheme[1]. Also, we prove the stability of this iteration. Throughout this paper the set of all positive integers and zero is shown by $\mathbb{N}$. Let $B$ be a Banach space, $C$ be a nonempty closed convex subset of $B$ and $T$ a self-map of $C$. An element $u^{*}$ of $C$ is called a fixed point of $T$ if and only if $T u^{*}=u^{*}$ [3]. The set of all fixed point of $T$ is denoted by $F_{T}$.

Recently, Badri [1] defined the following Jungck-Picard-S iterative scheme.

\section{Definition (1.1) [1]:}

Let $B$ be a Banach space and $C$ be a nonempty subset of $B$. Let $T, S: C \rightarrow C$ be two self mappings such that $T(C) \subseteq$ $S(C)$. For $x_{1} \in C$, the Jungck-Picard-S iterative scheme is the sequence $\left\{S x_{n}\right\}_{n=1}^{\infty}$ defined by

$S x_{n+1}=T y_{n}$

$S y_{n}=\left(1-\beta_{n}\right) T x_{n}+\beta_{n} T z_{n}$

$$
S z_{n}=\left(1-\gamma_{n}\right) S x_{n}+\gamma_{n} T x_{n} n \in \mathbb{N}
$$

where $\left\{\beta_{n}\right\}_{n=1}^{\infty}$ and $\left\{\gamma_{n}\right\}_{n=1}^{\infty}$ are real sequences in $[0,1)$ such that $\sum_{n=1}^{\infty} \beta_{n} \gamma_{n}=\infty$.
We have proved in [13] that the Jungck-Picard-S converges to a unique common fixed point when applied to a pair of Jungck-contraction mappings. Also, we have shown that the Jungck-Picard-S iterative scheme converges to the fixed point faster than each of Jungck-Noor [10], Junck-SP[4] and Jungck-CR [9] iterative schemes.

Definition (1.2) [8]: Let $B$ be a Banach space and $C$ be a nonempty subset of $B$. Let $T, S: C \rightarrow C$ be two self mappings such that $T(C) \subseteq S(C)$. The Jungck-contraction mapping is defined by:

$$
d(T x, T y) \leq \delta d(S x, S y), 0 \leq \delta<1
$$

\section{Stability}

Now we prove that Jungck-Picard-S iterative scheme (1.1) is stable with respect to $(S, T)$.

First we need the following definition:

\section{Definition (2.1) [11]:}

Let $(X, d)$ be a metric space and let $T, S: X \rightarrow X$ be self mappings. Let $z \in C(S, T)$ that is there exists $u^{*}$ such that $S z=T z=u^{*}$. For any $x_{1} \in X$, the sequence $\left\{S x_{n}\right\}_{n=1}^{\infty}$ generated by the general iterative procedure $x_{1} \in X$

$$
S x_{n+1}=f\left(T, x_{n}\right), n \in \mathbb{N}
$$

where $x_{0}$ is an initial approximation and $f\left(T, x_{n}\right)$ denotes to any iteration and suppose that it converges to $u^{*} \in X$. Let $\left\{S y_{n}\right\}_{n=0}^{\infty} \subset X$ be an arbitrary sequence and $\varepsilon_{n}=$ $d\left(S y_{n+1}, f\left(T, y_{n}\right)\right), n \in \mathbb{N}$. Then the iterative procedure $(2.1)$ is $(S, T)$-stable or stable with respect to $(S, T)$ if we have

$$
\lim _{n \rightarrow \infty} \varepsilon_{n}=0 \text { if and only if } \lim _{n \rightarrow \infty} S y_{n}=u^{*} .
$$

The following lemma will be needed in the next theorem.

\section{Lemma (2.2) [12]:}

Let $\left\{\tau_{n}\right\}_{n=1}^{\infty}$ and $\left\{\rho_{n}\right\}_{n=1}^{\infty}$ be nonnegative real sequences satisfying the following inequality:

$$
\tau_{n+1} \leq\left(1-\lambda_{n}\right) \tau_{n}+\rho_{n}
$$

where $\lambda_{n} \in(0,1)$ for all $n \geq n_{1}, \sum_{n=1}^{\infty} \lambda_{n}=\infty$ and $\frac{\rho_{n}}{\lambda_{n}} \rightarrow 0$ as $\mathrm{n} \rightarrow \infty$. Then $\lim _{n \rightarrow \infty} \tau_{n}=0$. 


\title{
International Journal of Science and Research (IJSR) \\ ISSN (Online): 2319-7064
}

Index Copernicus Value (2016): 79.57 | Impact Factor (2015): 6.391

Theorem (2.3):

Let $C$ be a nonempty closed convex subset of a Banach space $B, S, T: C \rightarrow C$ be two self-mappings satisfying Jungck-contraction condition (1.2) assume $T(C) \subseteq S(C)$, let $\left\{S x_{n}\right\}_{n=1}^{\infty}$ be the Jungck-Picard-S iterative scheme generated by (1.1) converges to $u^{*} \in F$ such that $\sum_{n=1}^{\infty} \beta_{n} \gamma_{n}=\infty$. Then the Jungck-Picard-S iterative scheme is stable with respect to $(S, T)$.

\section{Proof:}

Let $\left\{S x_{n}\right\}_{n=1}^{\infty}$ converges to $u^{*}$ and $\left\{S a_{n}\right\}_{n=1}^{\infty}$ be an arbitrary sequence in $C$.

Define $\varepsilon_{n}=\left\|S a_{n+1}-T b_{n}\right\|$ where

$$
\begin{aligned}
& S b_{n}=\left(1-\beta_{n}\right) T a_{n}+\beta_{n} T c_{n} \\
& S c_{n}=\left(1-\gamma_{n}\right) S a_{n}+\gamma_{n} T a_{n}
\end{aligned}
$$

Now for some $0 \leq \delta<1$ such that

$\|T x-T y\| \leq \delta\|S x-S y\|$ we get:

$\left\|S a_{n+1}-u^{*}\right\|=\left\|S a_{n+1}-T b_{n}+T b_{n}-u^{*}\right\|$

$$
\begin{aligned}
& \leq\left\|S a_{n+1}-T b_{n}\right\|+\left\|T b_{n}-u^{*}\right\| \\
& \leq \varepsilon_{n}+\left\|T b_{n}-u^{*}\right\| \\
& \leq \varepsilon_{n}+\delta\left\|S b_{n}-u^{*}\right\|
\end{aligned}
$$

On the other hand

$$
\begin{aligned}
\left\|S b_{n}-u^{*}\right\| & =\left\|\left(1-\beta_{n}\right) T a_{n}+\beta_{n} T c_{n}-u^{*}\right\| \\
& \leq\left(1-\beta_{n}\right)\left\|T a_{n}-u^{*}\right\|+\beta_{n}\left\|T c_{n}-u^{*}\right\|
\end{aligned}
$$

Also we get

$\left\|S c_{n}-u^{*}\right\| \leq\left(1-\beta_{n}\right) \delta\left\|S a_{n}-u^{*}\right\|+\beta_{n} \delta\left\|S c_{n}-u^{*}\right\|$

$$
\begin{aligned}
& =\left\|\left(1-\gamma_{n}\right) S a_{n}+\gamma_{n} T a_{n}-u^{*}\right\| \\
& \leq\left(1-\gamma_{n}\right)\left\|S a_{n}-u^{*}\right\|+\gamma_{n}\left\|T a_{n}-u^{*}\right\|
\end{aligned}
$$

Thus

$\left\|S c_{n}-u^{*}\right\|=\left[1-\gamma_{n}(1-\delta)\right]\left\|S a_{n}-u^{*}\right\|$

(2.4)

Substituting (2.3) and (2.4) in (2.2), we have

$\left\|S a_{n+1}-u^{*}\right\| \leq \varepsilon_{n}+\delta^{2}\left[\left(1-\beta_{n}\right)+\beta_{n}\left[1-\gamma_{n}(1-\right.\right.$

SSan-u*

$$
=\varepsilon_{n}+\delta^{2}\left[1-\beta_{n} \gamma_{n}(1-\delta)\right]\left\|S a_{n}-u^{*}\right\|
$$

By hypothesis we have $\lim _{n \rightarrow \infty} \varepsilon_{n}=0$ and $\beta_{n}, \gamma_{n}, \delta \in[0,1)$ then using Lemma (2.2) we get $\lim _{n \rightarrow \infty}\left\|S a_{n}-u^{*}\right\|=0$. Hence, we get $\lim _{n \rightarrow \infty} S a_{n}=u^{*}$.

Now suppose that $\lim _{n \rightarrow \infty} S a_{n}=u^{*}$ and we have to show that $\lim _{n \rightarrow \infty} \varepsilon_{n}=0$.

We have that

$$
\begin{aligned}
\varepsilon_{n} & =\left\|S a_{n+1}-T b_{n}\right\| \\
\leq & \left\|S a_{n+1}-u^{*}\right\|+\left\|T b_{n}-u^{*}\right\| \\
\leq & \left\|S a_{n+1}-u^{*}\right\|+\delta\left\|S b_{n}-u^{*}\right\| \\
= & \left\|S a_{n+1}-u^{*}\right\|+\delta\left\|\left(1-\beta_{n}\right) T a_{n}+\beta_{n} T c_{n}-u^{*}\right\| \\
\leq & \left\|S a_{n+1}-u^{*}\right\|+\delta\left(1-\beta_{n}\right)\left\|T a_{n}-u^{*}\right\|+ \\
\delta \beta_{n} & \left\|T c_{n}-u^{*}\right\| \\
\leq & \left\|S a_{n+1}-u^{*}\right\|+\left(1-\beta_{n}\right) \delta^{2}\left\|S a_{n}-u^{*}\right\|+ \\
\beta_{n} \delta^{2} & \left\|S c_{n}-u^{*}\right\| \\
= & \left\|S a_{n+1}-u^{*}\right\|+\left(1-\beta_{n}\right) \delta^{2}\left\|S a_{n}-u^{*}\right\| \\
& +\beta_{n} \delta^{2}\left\|\left(1-\gamma_{n}\right) S a_{n}+\gamma_{n} T a_{n}-u^{*}\right\| \\
\leq & \left\|S a_{n+1}-u^{*}\right\|+\left(1-\beta_{n}\right) \delta^{2}\left\|S a_{n}-u^{*}\right\| \\
& +\beta_{n} \delta^{2}\left(1-\gamma_{n}\right)\left\|S a_{n}-u^{*}\right\|+\beta_{n} \gamma_{n} \delta^{2}\left\|T a_{n}-u^{*}\right\| \\
\leq & \left\|S a_{n+1}-u^{*}\right\|+\left(1-\beta_{n} \gamma_{n}\right) \delta^{2}\left\|S a_{n}-u^{*}\right\|+ \\
\beta_{n} \gamma_{n} \delta^{3}\left\|S a_{n}-u^{*}\right\| & \\
= & \left\|S a_{n+1}-u^{*}\right\|+\delta^{2}\left[1-\beta_{n} \gamma_{n}(1-\delta)\right]\left\|S a_{n}-u^{*}\right\|
\end{aligned}
$$

By taking $n$ goes to infinity we get: $\lim _{n \rightarrow \infty} \varepsilon_{n}=\lim _{n \rightarrow \infty}\left\|S a_{n+1}-T b_{n}\right\|=0$. Then the Jungck-Picard-S iterative scheme (1.1) is stable with respect to $(S, T)$

\section{Data Dependence}

In the following theorem, we establish data dependence result of Jungck-Picard-S iterative scheme but first we need the following definition.

\section{Definition (3.1) [3]:}

Let $X$ be a Banach space, $(S, T),(\tilde{S}, \tilde{T}): X \rightarrow X$ be self mappings on $X$ such that $T(X) \subseteq S(X)$ and $\tilde{T}(X) \subseteq \tilde{S}(X)$. We say that the pair $(\tilde{S}, \tilde{T})$ is an approximate mapping pair of $(S, T)$ if for all $x \in X$ and for fixed $\varepsilon_{1}>0$ and $\varepsilon_{2}>0$, we have

$$
\|T x-\tilde{T} x\| \leq \varepsilon_{1},\|S x-\tilde{S} x\| \leq \varepsilon_{2}
$$

\section{Lemma (3.2), [11]:}

Let $\left\{a_{n}\right\}_{n=1}^{\infty}$ be a nonnegative sequence for which one assumes there exists $n_{1} \in \mathbb{N}$, such that for all $\mathrm{n} \geq n_{1}$ one has satisfied the inequality

$$
a_{n+1} \leq\left(1-\lambda_{n}\right) a_{n}+\lambda_{n} \rho_{n}
$$

where $\lambda_{n} \in(0,1)$, for all $\mathrm{n} \in \mathbb{N}, \sum_{n=1}^{\infty} \lambda_{n}=\infty$ and $\rho_{n} \geq 0$, for all $n \in \mathbb{N}$. Then the following inequality holds

\section{Theorem (3.3):}

$$
\lim _{n \rightarrow \infty} \operatorname{supa}_{n} \leq \lim _{n \rightarrow \infty} \sup _{n}
$$

Let $X$ be a Banach space and $(\tilde{S}, \tilde{T}): X \rightarrow X$, be an approximate mapping of the pair $(S, T): X \rightarrow X$ satisfying Jungck-contraction condition (1.2). Let $z \in C(S, T)$ and $\tilde{z} \in C(\tilde{S}, \tilde{T})$ be the coincidence points of $S, T$ and $\tilde{S}, \tilde{T}$ respectively that is $S z=T z=u^{*}$ and $\tilde{S} \tilde{z}=\tilde{T} \tilde{z}=\tilde{u}^{*}$. Let $\left\{S x_{n}\right\}_{n=1}^{\infty}$ be the Jungck-Picard-S iterative scheme generated by (1.1) with

1) $\frac{1}{2}<\beta_{n} \gamma_{n}$ for all $n \in \mathbb{N}$.

2) $\sum_{n=1}^{\infty} \beta_{n} \gamma_{n}=\infty$.

Let $\left\{\tilde{S} \tilde{x}_{n}\right\}_{n=1}^{\infty}$ be a sequence defined by

$\tilde{x}_{1} \in X$

$\tilde{S} \tilde{x}_{n+1}=\tilde{T} \tilde{y}_{n}$

$\tilde{S} \tilde{y}_{n}=\left(1-\beta_{n}\right) \tilde{T} x_{n}+\beta_{n} \tilde{T} \tilde{z}_{n}$

$$
\tilde{S} \tilde{z}_{n}=\left(1-\gamma_{n}\right) \tilde{S} \tilde{x}_{n}+\gamma_{n} \tilde{T} \tilde{x}_{n}, n \in \mathbb{N}
$$

Assume that $\left\{S x_{n}\right\}_{n=1}^{\infty}$ and $\left\{\tilde{S} \tilde{x}_{n}\right\}_{n=1}^{\infty}$ converges to $u^{*}$ and $\tilde{u}^{*}$ respectively.

Then we have

$$
\left\|u^{*}-\tilde{u}^{*}\right\| \leq \frac{5\left(\varepsilon_{1}+\varepsilon_{2}\right)}{1-\delta}
$$

Proof: It follows from Jungck-Picard-S iteration (1.1) and Jungck-contraction condition (1.2), (3.1) and (3.2) that

$$
\begin{aligned}
\left\|S x_{n+1}-\tilde{S} \tilde{x}_{n+1}\right\| & =\left\|T y_{n}-\tilde{T} \tilde{y}_{n}\right\| \\
& =\left\|T y_{n}-T \tilde{y}_{n}+T \tilde{y}_{n}-\tilde{T} \tilde{y}_{n}\right\| \\
& \leq\left\|T y_{n}-T \tilde{y}_{n}\right\|+\left\|T \tilde{y}_{n}-\tilde{T} \tilde{y}_{n}\right\|
\end{aligned}
$$

Thus

$$
\begin{gathered}
\left\|S x_{n+1}-\tilde{S} \tilde{x}_{n+1}\right\| \leq \varepsilon_{1}+\delta\left\|S y_{n}-S \tilde{y}_{n}\right\| \\
\leq \varepsilon_{1}+\delta\left\|S y_{n}-\tilde{S} \tilde{y}_{n}+\tilde{S} \tilde{y}_{n}-S \tilde{y}_{n}\right\| \\
\leq \varepsilon_{1}+\delta\left\|S y_{n}-\tilde{S} \tilde{y}_{n}\right\|+\delta\left\|\tilde{S} \tilde{y}_{n}-S \tilde{y}_{n}\right\| \\
\leq \varepsilon_{1}+\delta \varepsilon_{2}+\delta\left\|S y_{n}-\tilde{S} \tilde{y}_{n}\right\|
\end{gathered}
$$

\section{Volume 6 Issue 12, December 2017}

\author{
www.ijsr.net
}


But

$\left\|S y_{n}-\tilde{S} \tilde{y}_{n}\right\|=\|\left(1-\beta_{n}\right) T x_{n}+\beta_{n} T z_{n}-\left(1-\beta_{n}\right) \tilde{T} \tilde{x}_{n}+$ BnTZn

$$
\leq\left(1-\beta_{n}\right)\left\|T x_{n}-\tilde{T} \tilde{x}_{n}\right\|+\beta_{n}\left\|T z_{n}-\tilde{T} \tilde{z}_{n}\right\|
$$

Substitute (3.4) in (3.3) we get:

$$
\begin{aligned}
& \left\|S x_{n+1}-\tilde{S} \tilde{x}_{n+1}\right\| \leq \varepsilon_{1}+\delta \varepsilon_{2}+\delta\left(1-\beta_{n}\right)\left\|T x_{n}-\tilde{T} \tilde{x}_{n}\right\| \\
& \quad+\delta \beta_{n}\left\|T z_{n}-\tilde{T} \tilde{z}_{n}\right\| \\
& \quad=\varepsilon_{1}+\delta \varepsilon_{2}+\delta\left(1-\beta_{n}\right)\left\|T x_{n}-T \tilde{x}_{n}+T \tilde{x}_{n}-\tilde{T} \tilde{x}_{n}\right\| \\
& \quad+\delta \beta_{n}\left\|T z_{n}-T \tilde{z}_{n}+T \tilde{z}_{n}-\tilde{T} \tilde{z}_{n}\right\| \\
& \quad \leq \varepsilon_{1}+\delta \varepsilon_{2}+\delta\left(1-\beta_{n}\right)\left\|T x_{n}-T \tilde{x}_{n}\right\| \\
& \quad+\delta\left(1-\beta_{n}\right)\left\|T \tilde{x}_{n}-\tilde{T} \tilde{x}_{n}\right\|+\delta \beta_{n}\left\|T z_{n}-T \tilde{z}_{n}\right\| \\
& \quad+\delta \beta_{n}\left\|T \tilde{z}_{n}-\tilde{T} \tilde{z}_{n}\right\| \\
& \quad \leq \varepsilon_{1}+\delta \varepsilon_{2}+\delta\left(1-\beta_{n}\right) \varepsilon_{1}+\delta^{2}\left(1-\beta_{n}\right)\left\|S x_{n}-S \tilde{x}_{n}\right\| \\
& \quad+\beta_{n} \delta \varepsilon_{1}+\beta_{n} \delta^{2}\left\|S z_{n}-S \tilde{z}_{n}\right\| \\
& \quad+\varepsilon_{1}(1+\delta)+\delta \varepsilon_{2} \\
& \quad+\delta^{2}\left(1-\beta_{n}\right)\left\|S x_{n}-\tilde{S} \tilde{x}_{n}+\tilde{S} \tilde{x}_{n}-S \tilde{x}_{n}\right\| \\
& \quad+\beta_{n} \delta^{2}\left\|S z_{n}-\tilde{S} \tilde{z}_{n}+\tilde{S} \tilde{z}_{n}-S \tilde{z}_{n}\right\|
\end{aligned}
$$

Therefore

$$
\begin{aligned}
& \left\|S x_{n+1}-\tilde{S} \tilde{x}_{n+1}\right\| \leq \varepsilon_{1}(1+\delta)+\delta \varepsilon_{2}+\delta^{2}\left(1-\beta_{n}\right)\left\|S x_{n}-\tilde{S} \tilde{x}_{n}\right\| \\
& \quad+\delta^{2}\left(1-\beta_{n}\right)\left\|\tilde{S} \tilde{x}_{n}-S \tilde{x}_{n}\right\|+\beta_{n} \delta^{2}\left\|S z_{n}-\tilde{S} \tilde{z}_{n}\right\| \\
& \quad+\beta_{n} \delta^{2}\left\|\tilde{S} \tilde{z}_{n}-S \tilde{z}_{n}\right\| \\
& \quad \leq \varepsilon_{1}(1+\delta)+\delta \varepsilon_{2}+\delta^{2}\left(1-\beta_{n}\right) \varepsilon_{2}+\beta_{n} \delta^{2} \varepsilon_{2} \\
& \quad+\delta^{2}\left(1-\beta_{n}\right)\left\|S x_{n}-\tilde{S} \tilde{x}_{n}\right\|+\beta_{n} \delta^{2}\left\|S z_{n}-\tilde{S} \tilde{z}_{n}\right\| \\
& \quad=\varepsilon_{1}(1+\delta)+\delta(1+\delta) \varepsilon_{2} \\
& \quad+\delta^{2}\left(1-\beta_{n}\right)\left\|S x_{n}-\tilde{S} \tilde{x}_{n}\right\|+\beta_{n} \delta^{2}\left\|S z_{n}-\tilde{S} \tilde{z}_{n}\right\|(3.5)
\end{aligned}
$$

But

$$
\begin{aligned}
\| S z_{n}- & \tilde{S} \tilde{z}_{n}\|=\|\left(1-\gamma_{n}\right) S x_{n}+\gamma_{n} T x_{n}-\left(1-\gamma_{n}\right) \tilde{S} \tilde{x}_{n}+\gamma_{n} \tilde{T} \tilde{x}_{n} \| \\
& \leq\left(1-\gamma_{n}\right)\left\|S x_{n}-\tilde{S} \tilde{x}_{n}\right\|+\gamma_{n}\left\|T x_{n}-\tilde{T} \tilde{x}_{n}\right\| \\
& =\left(1-\gamma_{n}\right)\left\|S x_{n}-\tilde{S} \tilde{x}_{n}\right\|+\gamma_{n}\left\|T x_{n}-T \tilde{x}_{n}+T \tilde{x}_{n}-\tilde{T} \tilde{x}_{n}\right\| \\
& \leq\left(1-\gamma_{n}\right)\left\|S x_{n}-\tilde{S} \tilde{x}_{n}\right\|+\gamma_{n}\left\|T x_{n}-T \tilde{x}_{n}\right\| \\
& +\gamma_{n}\left\|T \tilde{x}_{n}-\tilde{T} \tilde{x}_{n}\right\| \\
& \leq\left(1-\gamma_{n}\right)\left\|S x_{n}-\tilde{S} \tilde{x}_{n}\right\|+\gamma_{n} \varepsilon_{1}+\gamma_{n} \delta\left\|S x_{n}-S \tilde{x}_{n}\right\| \\
& =\left(1-\gamma_{n}\right)\left\|S x_{n}-\tilde{S} \tilde{x}_{n}\right\|+\gamma_{n} \varepsilon_{1} \\
& +\gamma_{n} \delta\left\|S x_{n}-\tilde{S} \tilde{x}_{n}+\tilde{S} \tilde{x}_{n}-S \tilde{x}_{n}\right\| \\
& \leq\left(1-\gamma_{n}\right)\left\|S x_{n}-\tilde{S} \tilde{x}_{n}\right\|+\gamma_{n} \varepsilon_{1} \\
& +\gamma_{n} \delta\left\|S x_{n}-\tilde{S} \tilde{x}_{n}\right\|+\gamma_{n} \delta\left\|\tilde{S} \tilde{x}_{n}-S \tilde{x}_{n}\right\| \\
& \quad \leq\left[1-\gamma_{n}(1-\delta)\right]\left\|S x_{n}-\tilde{S} \tilde{x}_{n}\right\|+\gamma_{n} \varepsilon_{1}+\gamma_{n} \delta \varepsilon_{2} \text { (3.6) }
\end{aligned}
$$

Substituting (3.6) in (3.5), we obtain:

$\left\|S x_{n+1}-\tilde{S} \tilde{x}_{n+1}\right\| \leq \varepsilon_{1}(1+\delta)+\delta(1+\delta) \varepsilon_{2}$

$+\delta^{2}\left(1-\beta_{n}\right)\left\|S x_{n}-\tilde{S} \tilde{x}_{n}\right\|$

$+\beta_{n} \delta^{2}\left[\gamma_{n} \varepsilon_{1}+\gamma_{n} \delta \varepsilon_{2}+\left[1-\gamma_{n}(1-\delta)\right]\left\|S x_{n}-\tilde{S} \tilde{x}_{n}\right\|\right]$

$=\delta^{2}\left[1-\beta_{n} \gamma_{n}(1-\delta)\right]\left\|S x_{n}-\tilde{S} \tilde{x}_{n}\right\|$

$+\left(1+\delta+\beta_{n} \gamma_{n} \delta^{2}\right) \varepsilon_{1}+\delta\left(1+\delta+\beta_{n} \gamma_{n} \delta^{2}\right) \varepsilon_{2}$

$\leq\left[1-\beta_{n} \gamma_{n}(1-\delta)\right]\left\|S x_{n}-\tilde{S} \tilde{x}_{n}\right\|$

$+\left(1+\delta+\beta_{n} \gamma_{n} \delta^{2}\right) \varepsilon_{1}$

$$
+\delta\left(1+\delta+\beta_{n} \gamma_{n} \delta\right) \varepsilon_{2}
$$

Since $\beta_{n}, \gamma_{n} \in[0,1]$ and $\frac{1}{2}<\beta_{n} \gamma_{n}$ for all $n \in \mathbb{N}$ and using of the fact $\delta \in(0,1)$ in $(3.7)$

Yields:

$$
\begin{aligned}
\left\|S x_{n+1}-\tilde{S} \tilde{x}_{n+1}\right\| \leq[1 & \left.-\beta_{n} \gamma_{n}(1-\delta)\right]\left\|S x_{n}-\tilde{S} \tilde{x}_{n}\right\| \\
& +\beta_{n} \gamma_{n}(1-\delta)\left[\frac{5\left(\varepsilon_{1}+\varepsilon_{2}\right)}{1-\delta}\right]
\end{aligned}
$$

Put

$a_{n}=\left\|S x_{n}-\tilde{S} \tilde{x}_{n}\right\|$

$\lambda_{n}=\beta_{n} \gamma_{n}(1-\delta) \in(0,1)$ $\rho_{n}=\frac{5\left(\varepsilon_{1}+\varepsilon_{2}\right)}{1-\delta}$

Hence, the inequality (3.8)perform all assumptions in lemma (3.2) and thus an application of lemma (3.2) to (3.8)yields

$0 \leq \lim _{n \rightarrow \infty} \sup \left\|S x_{n}-\tilde{S} \tilde{x}_{n}\right\|$

$$
\leq \lim _{n \rightarrow \infty} \sup \frac{5\left(\varepsilon_{1}+\varepsilon_{2}\right)}{1-\delta}
$$

Since $\lim _{n \rightarrow \infty} S x_{n}=u^{*}$ and $\lim _{n \rightarrow \infty} \tilde{S} \tilde{x}_{n}=\tilde{u}^{*}$, then

$$
\left\|u^{*}-\tilde{u}^{*}\right\| \leq \frac{5\left(\varepsilon_{1}+\varepsilon_{2}\right)}{1-\delta}
$$

\section{References}

[1] Badri M., On a modified SP-iterative scheme approximation of fixed points, M.Sc. Thesis, College of Science, Baghdad University, 2016.

[2] Berinde V. (2003). On the approximation of fixed points of weak contractive mappings.Carpath. J. Math. 19: 7-22.

[3] Berinde V. 2007. Iterative Approximation of Fixed Points. Springer, Berlin.

[4] Chugh R., Kumar V., Strong convergence and stability results for Jungck-SP iterative scheme, International $\mathrm{J}$ of computer applications, vol. 36, no.12, (2011), pp:40-46.

[5] Harder A.M., Fixed point theory and stability results for fixed point iteration procedures, $\mathrm{Ph} . \mathrm{D}$. Thesis, University of Missouri-Rolla, Missouri, 1987.

[6] Harder A. M., Hicks T.L., A stable iteration procedure for nonexpansive mappings, Math. Japon. Vol. 33 (1988), pp.687-692.

[7] Harder A.M. and Hicks T.L., Stability results for fixed point iteration procedures, Math. Japon. Vol. 33(1988), pp.693-706.

[8] Jungck G., Commuting mappings and fixed points, Amer. Math. Monthly vol. 83, no. 4, (1976), pp:261263.

[9] Hussain N., Kumar V. and Kutbi M. A., On rate of convergence of Jungck-type iterative schemes, Hindawi Publishing Corporation Abstract and Applied Analysis, (2013), Article ID 132626, pp:1-15.

[10] Olatinwo M. O., A generalization of some convergence results using a Jungck-Noor three-step iteration process in arbitrary Banach space, Fasc. Math., vol.40, (2008), pp:37-43.

[11] Singh S. L. and Prasad B., Some concidence theorems and stability of iterative procedures, Comp. and Math. With Appl., vol.55, no.11, (2008), pp:2512-2520.

[12] Soltuz S. and Grosan T., Data dependence for Ishikawa iteration when dealing with contractive like operators. Fixed Point Theory and Appl. (2008), Article ID 242916, 7 pages.

[13]Zeana Z. J. and Assma K. A., On rate of convergence of Jungck-Picard-S iterative scheme, International Journal of Science and Research (IJSR), vol. 6, no. 5, (2017), pp:615-619. 